\title{
Enveloping algebras with just infinite Gelfand-Kirillov dimension
}

\author{
Natalia K. Iyudu and Susan J. Sierra
}

\begin{abstract}
Let $\mathfrak{g}$ be the Witt algebra or the positive Witt algebra. It is well known that the enveloping algebra $U(\mathfrak{g})$ has intermediate growth and thus infinite Gelfand-Kirillov (GK-) dimension. We prove that the GK-dimension of $U(\mathfrak{g})$ is just infinite in the sense that any proper quotient of $U(\mathfrak{g})$ has polynomial growth. This proves a conjecture of Petukhov and the second named author for the positive Witt algebra. We also establish the corresponding results for quotients of the symmetric algebra $S(\mathfrak{g})$ by proper Poisson ideals.

In fact, we prove more generally that any central quotient of the universal enveloping algebra of the Virasoro algebra has just infinite GK-dimension. We give several applications. In particular, we easily compute the annihilators of Verma modules over the Virasoro algebra.
\end{abstract}

\section{Introduction}

Let $\mathbb{K}$ be a field of characteristic zero, and let $W$ be the Witt algebra, which has $\mathbb{K}$-basis

$$
\left\{e_{n}: n \in \mathbb{Z}\right\}
$$

with the Lie bracket

$$
\left[e_{i}, e_{j}\right]=(j-i) e_{i+j}
$$

We let $W_{+}$, the positive Witt algebra, be the Lie subalgebra of $W$ spanned by $\left\{e_{n}: n \geqslant 1\right\}$.

The Witt algebra is a central quotient of the Virasoro algebra, Vir, which has $\mathbb{K}$-basis

$$
\left\{e_{n}: n \in \mathbb{Z}\right\} \cup\{c\}
$$

Key words and phrases: Witt algebra, positive Witt algebra, Virasoro algebra, GelfandKirillov dimension.

2010 Mathematics Subject Classification: primary 16S30, 17B68, 16P90; secondary 17 B65. 
and Lie bracket

$$
\left[e_{i}, e_{j}\right]=(j-i) e_{i+j}+\frac{i^{3}-i}{12} \delta_{i+j, 0} c, \quad c \text { central. }
$$

The Virasoro algebra and its representations are ubiquitous in conformal field theory.

These algebras were testing examples for the fundamental and important question of whether there is an infinite-dimensional Lie algebra with a (left and right) noetherian enveloping algebra. This question has been asked by many people, including Ralph Amayo and Ian Stewart [AS74, Question 27, p. 396], Ken Brown [Bro07, Question B], Jacques Dixmier, and Victor Latyshev, and conjecturally has a negative answer; see [SW14, Conjecture 0.1]. Recently it was shown by the second named author and Chelsea Walton [SW14] that the conjecture holds for the Lie algebras above: that is, $U\left(W_{+}\right), U(W)$, and $U(V i r)$ are not left or right Noetherian. The question is still unsolved in full generality.

However, the two-sided ideal structure of these enveloping algebras is extremely sparse, and it seems possible that they satisfy the ascending chain condition for two-sided ideals, a property sometimes known as being weakly Noetherian. Further, two-sided ideals of $W$ and $W_{+}$are known to be large, and Petukhov and the second named author have conjectured:

Conjecture 1.1. ([PS17, Conjecture 1.2]) The universal enveloping algebra $U\left(W_{+}\right)$has just infinite GK-dimension; that is, if $I$ is a non-zero ideal of $U\left(W_{+}\right)$, then $U\left(W_{+}\right) / I$ has polynomial growth.

(In this paper, we use polynomial growth as a synonym for finite GK-dimension.)

This conjecture was proved in [PS17] in the particular case that the ideal $I$ is generated by quadratic expressions in the $e_{i}$. In this paper we establish the conjecture in full, and generalise our arguments to prove that $U(W)$ and indeed any central factor of $U($ Vir $)$ has just infinite GK-dimension in the sense above. Our main result is:

Theorem 1.2. (See Theorems 3.2, 4.1 and 5.3) The algebras $U\left(W_{+}\right), U(W)$, and $U(V i r) /(c-\varkappa)$, for any $\varkappa \in \mathbb{K}$, have just infinite $G K$-dimension. In particular, Conjecture 1.1 holds.

We note that these algebras are all finitely generated and as many authors have noticed [KKM83], [Smi76] and [Ufn78] have intermediate growth (and thus infinite GK-dimension). The natural set of normal words is monomials of the form $e_{i_{1}}, \ldots, e_{i_{k}}, i_{1} \leqslant i_{2} \ldots \leqslant i_{k}$. Here the number of monomials with $i_{1}+i_{2}+\ldots+i_{k}=n$ is in fact the number of partitions of $n$, which was shown by Hardy to be bounded by $e^{c \sqrt{n}}$, an estimate later dramatically improved by Ramanujan. 
We also consider the induced Poisson structures on the symmetric algebras $S\left(W_{+}\right), S(W)$, and $S(V i r)$, and prove:

Theorem 1.3. (See Theorems 2.1 and 5.5) Let I be a proper Poisson ideal of $S\left(W_{+}\right)$. Then $S(W) / I$ has polynomial growth. Similar statements hold for $S(W)$ and for $S($ Vir $) /(c-\varkappa)$, for any $\varkappa \in \mathbb{K}$.

The Lie algebra Vir has a triangular decomposition, and so the classical notion of a Verma module makes sense. As an application of Theorem 1.2, we easily compute annihilators of Verma modules for Vir:

Theorem 1.4. (See Corollary 6.2) Let $M$ be a Verma module over Vir with central charge $\varkappa$. Then the annihilator of $M$ in $U(\operatorname{Vir})$ is the ideal $(c-\varkappa)$. As a result, for any $\varkappa \in \mathbb{K}$ the algebra $U($ Vir $) /(c-\varkappa)$ is primitive.

Theorem 1.4 is an unpublished result of Wallach [WS13].

We also obtain:

Proposition 1.5. (See Proposition 6.4) The algebras $U(W), U\left(W_{+}\right)$, and $U(V i r)$ all satisfy the ascending chain condition for completely prime ideals.

Proposition 1.6. (See Proposition 6.5) Let $U(\mathcal{W})$ be either $U\left(W_{+}\right), U(V i r)$, or $U(\operatorname{Vir}) /(c-\varkappa)$ for some $\varkappa \in \mathbb{K}$. Then any epimorphism of $U(\mathcal{W})$ is an isomorphism.

This answers a question of Rowen and Small [RS17, Section 4].

The Witt algebra $W$ is a simple, graded, Lie algebra of polynomial growth. Such algebras were famously classified by Mathieu [Mat92]. It is interesting to ask which of these Lie algebras have enveloping algebras with just infinite GKdimension. This is the subject of ongoing research.

Methods. As notation, we will write the symmetric algebra $S\left(W_{+}\right)=\mathbb{K}\left[x_{1}\right.$, $\left.x_{2}, \ldots\right]$, where $x_{i}$ corresponds to $e_{i} \in W_{+}$. Likewise,

$$
S(W)=\mathbb{K}\left[\ldots, x_{-1}, x_{0}, x_{1}, \ldots\right] \quad \text { and } \quad S(\operatorname{Vir})=\mathbb{K}\left[\ldots, x_{-1}, x_{0}, x_{1}, \ldots, c\right] .
$$

The main idea in the proofs of both Theorems 1.2 and 1.3 is to show that if $g$ is a nonzero element of $S\left(W_{+}\right)$or $S(W)$, then for 'almost all' monomials $m$ in the $x_{i}$ (more precisely, for long enough monomials on big enough letters), the Poisson ideal generated by $g$ contains an element with leading term $m$. (See Lemma 2.2.) In the same way, we show that if $g$ is a nonzero element of $U\left(W_{+}\right)$or $U(W)$, then for almost all monomials $m$ in the variables $e_{i}$, the two-sided ideal generated by $g$ contains an element with leading term $m$. (See Lemmata 3.1 and 4.4.)

We summarise the argument for $S\left(W_{+}\right)$. Let $I$ be the Poisson ideal generated by $0 \neq g \in S\left(W_{+}\right)$. We introduce the following ordering on monomials in the $x_{i}$. 
Denote by $\operatorname{deg}(m)=i_{1}+\ldots+i_{k}$ the degree of a monomial $m=x_{i_{1}} \ldots x_{i_{k}}$, and let the length of $m$ be $|m|=\left|x_{i_{1}} \ldots x_{i_{k}}\right|=k$.

Then the ordering on the set of commutative monomials in the $x_{i}$ is defined as follows. For two monomials $m_{1}$ and $m_{2}$, we write $m_{1}<m_{2}$ if

- $\left|m_{1}\right|<\left|m_{2}\right|$ or

- $\left|m_{1}\right|=\left|m_{2}\right|$ and $\operatorname{deg}\left(m_{1}\right)<\operatorname{deg}\left(m_{2}\right)$ or

- $\left|m_{1}\right|=\left|m_{2}\right|, \operatorname{deg}\left(m_{1}\right)=\operatorname{deg}\left(m_{2}\right)$ and $m_{1}$ is less than $m_{2}$ with respect to the left-to-right lexicographical order when both $m_{1}$ and $m_{2}$ are written in increasing order: $m_{1}=x_{i_{1}} \ldots x_{i_{k}}, m_{2}=x_{j_{1}} \ldots x_{j_{k}}$ with $i_{1} \leqslant i_{2} \leqslant \ldots \leqslant i_{k}, j_{1} \leqslant j_{2} \leqslant \ldots \leqslant j_{k}$.

We show in Lemma 2.2 that all sufficiently long monomials on sufficiently 'big' letters can be written, modulo $I$, as a sum of smaller monomials. Here a letter is called big if it is bigger than $n=\left\{\max (2 i+1) \mid x_{i}\right.$ occurs in $\left.g\right\}$. By this means we are able to introduce a 'normal form' for monomials from $S\left(W_{+}\right)$. Namely, any element of $S\left(W_{+}\right)$can be written, modulo $I$, as a linear combination of monomials of the form $m=u v$, where $u$ is a monomial on the finite set of letters $x_{1}, \ldots x_{n-1}$, and $v$ is a monomial of restricted length on the set of big letters. A similar normal form works for $U\left(W_{+}\right)$.

In the case of $W$ (more generally, central quotients of Vir) the normal form is slightly different. Let $0 \neq J \triangleleft U(W)$. Any element of $U(W)$ can be written, modulo $J$, as a linear combination of monomials $m=u_{1} v u_{2}$, where $v$ is a monomial on the finite set of letters $e_{1-n}, \ldots e_{n-1}, u_{1}$ is a monomial of bounded length on letters smaller than $e_{-n}$, and $u_{2}$ is a monomial of bounded length on letters larger than $e_{n}$.

Counting the growth of these normal monomials gives us a polynomial estimate which bounds the growth of the quotient algebra. In the case of the full Witt algebra the growth counting is somewhat more involved, since the usual degree function $\operatorname{deg}(u)=i_{1}+\ldots+i_{k}$ will not supply us a finite filtration on $U(W) / J$. See Section 4.2 for the details of how this issue is resolved.

Notation. Throughout we fix the following notation. We denote the set of nonnegative integers by $\mathbb{N}$. If $R$ is a ring and $g \in R$, the two-sided ideal generated by $g$ is denoted $(g)$. If $R$ is a Poisson algebra, the Poisson ideal generated by $g$ is denoted $\{(g)\}$.

Let $S(\mathcal{W})$ denote either $S\left(W_{+}\right), S(\operatorname{Vir})$, or $S(\operatorname{Vir}) /(c-\varkappa)$ for some $\varkappa \in \mathbb{K}$. Likewise, let $U(\mathcal{W})$ be either $U\left(W_{+}\right), U(\operatorname{Vir})$, or $U(\operatorname{Vir}) /(c-\varkappa)$. Our convention is that $e_{1}, e_{2}, \ldots$ denote elements of $U(\mathcal{W})$, and that $x_{1}, x_{2}, \ldots$ are the corresponding elements of $S(\mathcal{W})$. Monomials $x_{i_{1}} x_{i_{2}} \ldots x_{i_{k}} \in S(\mathcal{W})$ are usually written in increasing order (if not specified otherwise), that is $i_{1} \leqslant i_{2} \leqslant \ldots \leqslant i_{k}$. The same shape $e_{i_{1}} e_{i_{2}} \ldots e_{i_{k}}$, where $i_{1} \leqslant i_{2} \leqslant \ldots \leqslant i_{k}$, of a monomial is considered normal in $U(\mathcal{W})$, and such a monomial is called standard. 


\section{The symmetric algebra of the positive Witt algebra}

Our main goal in this section is to prove the following theorem, which gives the first statement of Theorem 1.3:

Theorem 2.1. Let $I$ be a nonzero Poisson ideal of $S\left(W_{+}\right)$. Then $A=S\left(W_{+}\right) / I$ has polynomial growth.

Let $X=\left\{x_{1}, x_{2}, \ldots\right\}$, so $S\left(W_{+}\right)=\mathbb{K}[X]$, with Poisson bracket induced by defining $\left\{x_{i}, x_{j}\right\}=(j-i) x_{i+j}$. We equip $S\left(W_{+}\right)$with the degree grading given by setting $\operatorname{deg}\left(x_{j}\right)=j$. Thus the degree of a monomial $m=x_{i_{1}} \ldots x_{i_{k}}$ is $\operatorname{deg}(m)=i_{1}+\ldots+i_{k}$. Of course, we have the natural grading by the length of monomials as well, which we write as $\left|x_{i_{1}} \ldots x_{i_{k}}\right|=k$.

The key technique in the proof is the following reduction formula for elements of $A=S\left(W_{+}\right) / I$ in the case that $I=\{(g)\}$ is the Poisson ideal generated by a single, nonzero, degree-homogeneous polynomial $g$.

Lemma 2.2. Let $I=\{(g)\}$ be the Poisson ideal in $\mathbb{K}[X]=S\left(W_{+}\right)$generated by a nonzero degree-homogeneous polynomial $g \in \mathbb{K}[X]$. There exist positive integers $k$ and $n$ such that every monomial $m=x_{j_{1}} \ldots x_{j_{k}}$ such that $j_{\ell} \geqslant n$ for $1 \leqslant \ell \leqslant k$ satisfies

$$
m=h+\sum c_{s} m_{s}
$$

where $h \in I$ is degree-homogeneous with $\operatorname{deg}(h)=\operatorname{deg}(m)$, the sum is finite, $c_{s} \in \mathbb{K}$, and the $m_{s}$ are monomials of degree $\operatorname{deg}(m)$ such that for each $s$, either $\left|m_{s}\right|<k$ or $\left|m_{s}\right|=k$ and $i<n$ for at least one of the letters $x_{i}$ featuring in $m_{s}$.

To prove Lemma 2.2, we introduce the following two orderings on the set $[X]$ of commutative monomials in $X$. For two monomials $m_{1}$ and $m_{2}$, we write $m_{1}<m_{2}$ if

- $\left|m_{1}\right|<\left|m_{2}\right|$ or

- $\left|m_{1}\right|=\left|m_{2}\right|$ and $\operatorname{deg}\left(m_{1}\right)<\operatorname{deg}\left(m_{2}\right)$ or

- $\left|m_{1}\right|=\left|m_{2}\right|, \operatorname{deg}\left(m_{1}\right)=\operatorname{deg}\left(m_{2}\right)$ and $m_{1}$ is less than $m_{2}$ with respect to the left-to-right lexicographic order when both $m_{1}$ and $m_{2}$ are written in increasing order: $m_{1}=x_{i_{1}} \ldots x_{i_{k}}, m_{2}=x_{j_{1}} \ldots x_{j_{k}}$ with $i_{1} \leqslant i_{2} \leqslant \ldots \leqslant i_{k}, j_{1} \leqslant j_{2} \leqslant \ldots \leqslant j_{k}$.

Similarly, we write $m_{1} \prec m_{2}$ if

- $\left|m_{1}\right|<\left|m_{2}\right|$ or

- $\left|m_{1}\right|=\left|m_{2}\right|$ and $\operatorname{deg}\left(m_{1}\right)<\operatorname{deg}\left(m_{2}\right)$ or

- $\left|m_{1}\right|=\left|m_{2}\right|, \operatorname{deg}\left(m_{1}\right)=\operatorname{deg}\left(m_{2}\right)$ and $m_{1}$ is less than $m_{2}$ with respect to the left-to-right lexicographical order when both $m_{1}$ and $m_{2}$ are written in decreasing order: $m_{1}=x_{i_{1}} \ldots x_{i_{k}}, m_{2}=x_{j_{1}} \ldots x_{j_{k}}$ with $i_{k} \leqslant i_{k-1} \leqslant \ldots \leqslant i_{1}, j_{k} \leqslant j_{k-1} \leqslant \ldots \leqslant j_{1}$. (Equivalently, we may write $m_{1}$ and $m_{2}$ in increasing order and compare them with the right-to-left lexicographic order.) 
Note, that in the ordering < we compare the smallest letters first, and in the ordering $\prec$ we compare the biggest letters first. It is easy to see that both orderings are well-orderings on $[X]$ compatible with multiplication.

Proof of Lemma 2.2. Our ordering < satisfies the descending chain condition, and thus Lemma 2.2 can easily be obtained by repeated application of the following sublemma:

Sublemmma. There exist positive integers $k$ and $n$ such that every monomial $m \in[X]$ of length $k$ with $m \geqslant x_{n}^{k}$ satisfies

$$
m=h+\sum c_{s} m_{s}
$$

where $h \in I$ is degree-homogeneous with $\operatorname{deg}(h)=\operatorname{deg}(m)$, the sum is finite, $c_{s} \in \mathbb{K}$, and the $m_{s}$ are monomials of degree $\operatorname{deg}(m)$ such that $m_{s}<m$ for each $s$.

To prove the sublemma, let $\bar{g}$ be the leading monomial of $g$ with respect to $\prec$, and let $k=|\bar{g}|=|g|$. Without loss of generality we can assume that $\bar{g}$ features in $g$ with coefficient 1 . We write $\bar{g}$ in an increasing way: $\bar{g}=x_{i_{1}} \ldots x_{i_{k}}$ with $i_{1} \leqslant i_{2} \leqslant \ldots \leqslant i_{k}$. Pick any positive integer $n$ such that $n \geqslant 2 i_{k}+1$. We shall show that these $n$ and $k$ satisfy the required conditions.

Let $m \in[X]$ of length $k$ be such that $m \geqslant x_{n}^{k}$. Then $m=x_{j_{1}} \ldots x_{j_{k}}$ with $n \leqslant j_{1} \leqslant$ $\ldots \leqslant j_{k}$. If $a \in \mathbb{Z}_{\geqslant 1}$, let $d_{a}: \mathbb{K}[X] \rightarrow \mathbb{K}[X]$ be the derivation defined by $d_{a}(u)=\left\{u, x_{a}\right\}$, extending via the Leibniz rule. Note that $d_{a}$ is a graded derivation: if applied to a degree-homogeneous polynomial $f$, then $d_{a}(f)$ is degree-homogeneous of degree $\operatorname{deg}\left(d_{a}(f)\right)=\operatorname{deg}(f)+a$.

Consider

$$
h=d_{j_{1}-i_{k}} d_{j_{2}-i_{k-1}} \ldots d_{j_{k}-i_{1}}(g) .
$$

Since $g \in I$ and $I$ is a Poisson ideal, $h \in I$. Further, as the $d_{a}$ are graded and $g$ is degree-homogenous, so is $h$. The proof will be complete if we verify that

$$
h=c m+\sum c_{s} m_{s}
$$

where $c \neq 0, c_{s} \in \mathbb{K}$, and the $m_{s}$ are monomials such that $m_{s}<m$ for each $s$.

Let us apply the sequence of derivations $d_{j_{1}-i_{k}} d_{j_{2}-i_{k-1}} \ldots d_{j_{k}-i_{1}}$ to a monomial $u$ occurring in $g$. By the Leibniz rule we get a sum of monomials with coefficients obtained by prescribing which of the derivations acts on each letter of $u$.

Note (assuming that $d_{a}(u) \neq 0$ ) that $d_{a}(u)$ has the same length as $u$. Thus monomials in $h$ obtained from monomials in $g$ of length $<k$ are themselves of length $<k$ and therefore are smaller than $m$ with respect to $<$.

Suppose now that $u$ has length $k$. Then there are two options: either different differentials act on letters in different places in the monomial $u$ or this is not the case. 
We call the first of these ways permutational and the second non-permutational. Monomials in $h$ obtained from $u$ in a non-permutational way will have at least one letter unchanged and therefore will have at least one letter $x_{i}$ with $i \leqslant i_{k}<n$. Hence such monomials of $h$ are again smaller than $m$ with respect to $<$.

It remains to consider monomials of $h$ obtained from monomials in $g$ of length $k$ in a permutational way. For each monomial $u=x_{p_{1}} \ldots x_{p_{k}}$ with $p_{1} \leqslant \ldots \leqslant p_{k}$ occurring in $g$ and each permutation $\sigma \in \mathfrak{S}_{k}$, we obtain a monomial $w$ of $h$ given by

$$
w=x_{j_{1}-i_{k}+p_{\sigma(k)}} \ldots x_{j_{k}-i_{1}+p_{\sigma(1)}},
$$

occurring with coefficient

$$
\prod_{\ell=1}^{k}\left(j_{\ell}-i_{k-\ell+1}-p_{\sigma(k-\ell+1)}\right) .
$$

Since $u \preceq \bar{g}$, thus $p_{\sigma(k)} \leqslant p_{k} \leqslant i_{k}$. Hence $j_{1}-i_{k}+p_{\sigma(k)} \leqslant j_{1}$ and the equality $j_{1}-i_{k}+$ $p_{\sigma(k)}=j_{1}$ holds if and only if $p_{\sigma(k)}=p_{k}=i_{k}$. If $j_{1}-i_{k}+p_{\sigma(k)}<j_{1}$, then $w<m$ and we are done. If $j_{1}-i_{k}+p_{\sigma(k)}=j_{1}$, we have $p_{\sigma(k)}=p_{k}=i_{k}$. Since $u \preceq \bar{g}$ and $p_{\sigma(k)}=$ $p_{k}=i_{k}$, we have $p_{\sigma(k-1)} \leqslant p_{k-1} \leqslant i_{k-1}$. Hence $j_{2}-i_{k-1}+p_{\sigma(k-1)} \leqslant j_{2}$ and the equality $j_{2}-i_{k-1}+p_{\sigma(k-1)}=j_{2}$ holds if and only if $p_{\sigma(k-1)}=p_{k-1}=i_{k-1}$. Repeating the procedure, we see that $w \leqslant m$ and that $w=m$ only if $u=\bar{g}$ and the permutation $\sigma$ satisfies $i_{\sigma(s)}=i_{s}$ for $1 \leqslant s \leqslant k$.

Now, for each such $\sigma$, since $n \geqslant 2 i_{k}+1$, we claim that the coefficient of the monomial $m$ is a positive integer. Indeed, the coefficient is a product of factors of the form $j_{\ell}-i_{k-\ell+1}-p_{\sigma(k-\ell+1)}$, which are positive since $i_{k-\ell+1}+p_{\sigma(k-\ell+1)} \leqslant 2 i_{k}<n \leqslant j_{\ell}$. Thus the coefficient with which $m$ occurs in $h$ is nonzero. The other monomials in $h$ are $<m$. This completes the proof of the sublemma and thus of the lemma.

Note that we used in this proof only that $I$ is a module over $\mathbb{K}$ defined by the bracket multiplication (in other words a submodule of $S\left(W_{+}\right)$under the adjoint action of $\left.W_{+}\right)$.

We now prove Theorem 2.1. The key point of the proof is that, thanks to Lemma 2.2, $A=S\left(W_{+}\right) / I$ is spanned by the set of monomials $m$ in $[X]$ which admit a factorization $m=m_{1} m_{2}$, where $m_{1}$ is a monomial in $x_{1}, x_{2}, \ldots, x_{n-1}$ and $\left|m_{2}\right|<k$. Thus to estimate the growth of $A$ it suffices to count such monomials.

Proof of Theorem 2.1. As $S\left(W_{+}\right)$is finitely graded by degree, it is standard (see [KL00, Proposition 6.6]) that it suffices to show that $S\left(W_{+}\right) / I$ has polynomial growth if $I$ is a nonzero degree-graded Poisson ideal, and it clearly suffices to consider the case that $I$ is the Poisson ideal generated by a single nonzero degree-homogenous element $g$. Let $k$ and $n$ be the numbers produced by apply- 
ing Lemma 2.2 to $g$, and let $S$ be the set of all monomials $m$ in $[X]$ which admit a factorisation $m=m_{1} m_{2}$, where $m_{1}$ is a monomial in $x_{1}, x_{2}, \ldots, x_{n-1}$ and $\left|m_{2}\right|<k$.

By Lemma 2.2, each monomial $m \in[X] \backslash S$ can be written, modulo $I$, as a linear combination of monomials of degree $\operatorname{deg}(m)$, each of which either has length strictly less than $|m|$ or has length $|m|$ and features strictly fewer $x_{i}$ with $i \geqslant n$. Applying this observation repeatedly, we see that every monomial $m \in[X] \backslash S$ can be written, modulo $I$, as a linear combination of monomials from $S$ of the same degree as $m$. We will call such presentation a normal form of $m$. Hence the image of $S$ in $A=\mathbb{K}[X] / I$ spans $A$, and for fixed $N \in \mathbb{N}$, the number of monomials in $S$ of degree not exceeding $N$ provides an upper bound for $\operatorname{dim}\{u \in A \mid \operatorname{deg} u \leqslant N\}$. As $A$ is finitely $\mathbb{N}$-graded by degree, it is standard that the growth of this dimension bounds GKdim $A$.

It remains to estimate the number $p(N)$ of elements of $S$ of degree at most $N$. Clearly $p(N) \leqslant q(N) r(N)$, where $q(N)$ is the number of monomials in $x_{1}, \ldots, x_{n-1}$ of degree at most $N$ and $r(N)$ is the number of monomials in $x_{1}, x_{2}, \ldots$ of degree at most $N$ and length at most $k-1$. First, $q(N)$ does not exceed the number of monomials in $x_{1}, \ldots, x_{n-1}$ of length at most $N$, which is $\left(\begin{array}{c}N+n \\ N\end{array}\right)$. Thus there is a positive constant $c$ so that $q(N) \leqslant c N^{n}$ for all $N$. On the other hand, in a degree at most $N$ monomial of length at most $k-1$ in $x_{n}, x_{n+1}, \ldots$ there are no more than $N$ options for each letter and therefore $r(N) \leqslant N^{k-1}$ for all $N$. Hence $p(N) \leqslant c N^{k+n-1}$.

Hence $\operatorname{GKdim}(A) \leqslant k+n-1$, and $A$ has polynomial growth, as required.

We note that Theorem 2.1 is also proved in [PS17] (see Corollary 2.13), with a much less constructive proof.

To end the section, we fix a positive integer $k$ and consider the symmetric power $S^{k}\left(W_{+}\right)$. Let $g \in S^{k}\left(W_{+}\right)$be a nonzero degree-homogeneous element, and let $I$ be the $W_{+}$-submodule of $S^{k}\left(W_{+}\right)$generated by $g$. As noted after the proof of Lemma 2.2, the reduction formula in Lemma 2.2 still applies to $S^{k}\left(W_{+}\right) / I$, and the argument in the proof of Theorem 2.1 now gives that $\operatorname{GKdim} S^{k}\left(W_{+}\right) / I \leqslant k-1$. As $S^{k}\left(W_{+}\right)$clearly has GK-dimension $k$, we obtain:

Proposition 2.3. As a $W_{+}$-module, $S^{k}\left(W_{+}\right)$is $G K k$-critical.

For $k=2$, this was shown in [PS17, Corollary 4.15].

\section{The universal enveloping algebra of the positive Witt algebra}

That $U\left(W_{+}\right)$has just infinite GK-dimension follows from Theorem 2.1 using the Poisson GK-dimension defined in [PS17] and [PS17, Theorem 3.19]. However, a direct proof, which we give here, is also straightforward; the techniques of Section 2 apply also to $U\left(W_{+}\right)$. 
We begin by giving a noncommutative version of the reduction formula of Lemma 2.2. Our result is more general than needed here, for later use when considering quotients of $U(W)$.

By the Poincaré-Birkhoff-Witt theorem, $U(W)$ has a basis of monomials $e_{i_{1}} e_{i_{2}} \ldots e_{i_{k}}$ with $i_{1} \leqslant i_{2} \leqslant \ldots \leqslant i_{k}$. We call such monomials standard.

Lemma 3.1. Let $0 \neq g \in U(W)$, and let $I=U\left(W_{+}\right) g U\left(W_{+}\right)$be the $U\left(W_{+}\right)$-subbimodule of $U(W)$ generated by $g$. Then there exist positive integers $k$ and $n$ and an integer $\ell$ so that every standard monomial $m=e_{j_{1}} \ldots e_{j_{k}}$ with $n \leqslant j_{1} \leqslant \ldots \leqslant j_{k}$ satisfies

$$
m=h+\sum c_{t} m_{t}
$$

where $h \in I$, the sum is finite, $c_{t} \in \mathbb{K}$, and the $m_{t}$ are standard monomials so that for each $t$, we have $i \geqslant \ell$ for all letters $e_{i}$ featuring in $m_{t}$, and either $\left|m_{t}\right|<k$ or $\left|m_{t}\right|=k$ and $i<n$ for at least one of the letters featuring in $m_{t}$. Further, if $h$ is degree-homogeneous, then $\operatorname{deg}(m)=\operatorname{deg}(h)=\operatorname{deg}\left(m_{t}\right)$ for all $t$.

Proof. Let $k=|g|$. Writing $g$ as a sum of standard monomials, let $e_{\ell}$ be the smallest letter occurring in $g$ and define $n^{\prime}$ so that $e_{n^{\prime}}$ is the largest letter in $g$. Let $n=2\left|n^{\prime}\right|+1$.

There are well-defined monomial orderings $<$ and $\prec$ on standard monomials in $U(W)$, defined just as the corresponding orderings on commutative monomials $x_{i_{1}} \ldots x_{i_{k}}$ in the previous section. Note that $<$ does not satisfy the descending chain condition because $W$ has no least element, but the induced order on standard monomials in letters $\geqslant \ell$ does satisfy d.c.c. Thus, as in the proof of Lemma 2.2, it suffices to show that we can rewrite $m$, modulo $I$, as a linear combination of standard monomials in letters $\geqslant \ell$, each of which are $<m$.

For any $a \in \mathbb{Z}$, let $\partial_{a}=\left[\ldots, e_{a}\right]$ as a linear operator from $U(W) \rightarrow U(W)$. Recall that length defines a filtration on $U(W)$ whose associated graded ring is $S(W)$; for $f \in U(W)$ let $\operatorname{gr}(f)$ be the element of $S(W)$ associated to $f$, so $x_{i}=\operatorname{gr}\left(e_{i}\right)$. For any $p \in U(W)$ and any $a \in \mathbb{Z}$, we have

$$
\operatorname{gr} \partial_{a}(p)=d_{a}(\operatorname{gr}(p)) \quad \text { if } d_{a}(\operatorname{gr}(p)) \neq 0,
$$

where $d_{a}=\left\{\ldots, x_{a}\right\}$ as in the proof of Lemma 2.2.

Let $\bar{g}$ be the $\prec$-leading standard monomial in $g$ and write $\bar{g}=e_{i_{1}} \ldots e_{i_{k}}$ with $i_{1} \leqslant \ldots \leqslant i_{k}$. Let

$$
h=\partial_{j_{1}-i_{k}} \partial_{j_{2}-i_{k-1}} \ldots \partial_{j_{k}-i_{1}}(g) .
$$

Since for any letter $x_{p}$ featuring in $\operatorname{gr}(g)$, and for any $d_{a}$ which is applied to $x_{p}$, by our choice of $n$ we have $a>p$, as in the proof of Lemma 2.2. Thus, just as in that proof, and using (3.2),

$$
\operatorname{gr}(h)=d_{j_{1}-i_{k}} d_{j_{2}-i_{k-1}} \ldots d_{j_{k}-i_{1}}(\operatorname{gr}(g))=c \operatorname{gr}(m)+\sum c_{t} \operatorname{gr}\left(m_{t}^{\prime}\right),
$$


where $c \neq 0$, the sum is finite, $c_{t} \in \mathbb{K}$, and the $m_{t}^{\prime}$ are standard monomials so that $m_{t}^{\prime}<m$ for all $t$. Thus the length of $h-m-\sum c_{t} m_{t}^{\prime}$ is strictly smaller than $k$, so $h-m$ is a linear combination of standard monomials which are all strictly $<m$.

Since $a>0$ for all $\partial_{a}$ we have applied, only letters $\geqslant \ell$ occur in $h$. Finally, as the $\partial_{a}$ are graded linear maps, if $g$ is degree-homogenous so is $h$.

Theorem 3.2. Let $I$ be a nonzero two-sided ideal in $U\left(W_{+}\right)$. Then $A=$ $U\left(W_{+}\right) / I$ has polynomial growth.

Proof. All essential points of the proof occur the proof of Theorem 2.1.

As before, since $U\left(W_{+}\right)$is finitely graded by degree, we may assume that $I=(g)$ is the ideal generated by a single nonzero degree-homogenous element $g$. Let $k=|g|$.

Let $S$ be the set of standard monomials $m$ which admit a factorisation $m=$ $m_{1} m_{2}$, where $m_{1}$ is a standard monomial in $e_{1}, \ldots, e_{n-1}$ and $\left|m_{2}\right|<k$. It follows from the reduction formula in Lemma 3.1 that $U\left(W_{+}\right) / I$ is spanned by the image of $S$. The same counting argument as in the proof of Theorem 2.1 shows that $U\left(W_{+}\right) / I$ has polynomial growth.

Theorem 3.2 gives the first part of Theorem 1.2, dealing with $U\left(W_{+}\right)$.

\section{The enveloping algebra of the full Witt algebra}

In this section, we consider the enveloping algebra of the full Witt algebra, and show that it has just infinite GK-dimension. It clearly suffices to show:

Theorem 4.1. Let $I=(g)$ be a two-sided ideal in $U(W)$ generated by one nonzero element $g \in U(W)$. Then $A=U(W) / I$ has polynomial growth.

Throughout the section, we fix the meanings of $g, I$, and $A$ as in the statement of Theorem 4.1. Let $\pi: U(W) \rightarrow A$ be the natural map.

Now, $U(W)$ is finitely generated, say by $\left\{e_{-2}, e_{-1}, e_{1}, e_{2}\right\}$, and thus so is $A$. Since the growth of $A$ is controlled by the growth of any finite filtration, we are free to choose one that is convenient, but it will be a little bit more complicated this time to choose the right one. The problem is that unlike the situation for $W_{+}$, the usual degree function $\operatorname{deg}\left(e_{i}\right)=i$ does not give us a finite grading on $U(W)$; note that in the proofs of Theorems 2.1 and 3.2, the finiteness of the degree grading played a crucial role. Moreover, although of course there are many finite filtrations on $U(W)$, it is not necessarily clear how to choose one which induces a filtration on the quotient with polynomial growth.

Thus we will need to find an appropriate degree function which will give us a well-controlled finite filtration on $A$. We will see that a degree function of the form $\delta_{C}$, defined by $\delta_{C}\left(e_{i_{1}} \ldots e_{i_{k}}\right)=\left|i_{1}\right|+\ldots+\left|i_{k}\right|+C$, where $C$ is a constant, does the job. 


\subsection{A spanning set for $A$}

Our first step is to construct a set of standard monomials in $U(W)$ whose images span $A$.

Symmetrically to Lemma 3.1, we have:

Lemma 4.2. There exist positive integers $k$ and $n$ and an integer $\ell$ such that every standard monomial $m=e_{j_{1}} \ldots e_{j_{k}}$ with $j_{1} \leqslant \ldots \leqslant j_{k} \leqslant-n$ satisfies

$$
m=h+\sum c_{t} m_{t}
$$

where $h \in I$, the sum is finite, $c_{t} \in \mathbb{K}$, and the $m_{t}$ are standard monomials so that for each $t$, we have $i \leqslant \ell$ for all letters $e_{i}$ featuring in $m_{t}$, and either $\left|m_{t}\right|<k$ or $\left|m_{t}\right|=k$ and $i>-n$ for at least one of the letters featuring in $m_{t}$.

Proof. Consider the automorphism $\Phi$ of $U(W)$ defined by $\Phi\left(x_{i}\right)=-x_{-i}$ for $i \in \mathbb{Z}$. If we apply Lemma 3.1 to the ideal $\Phi(I)$ and the monomial $\pm \Phi(m)$, we obtain that $\Phi(m)=h+\sum c_{t} m_{t}$, where $h \in I$, the sum is finite, $c_{t} \in \mathbb{K}$, and the $m_{t}$ are standard monomials so that for each $t$, we have $i \geqslant \ell$ for all letters $e_{i}$ featuring in $m_{t}$, and either $\left|m_{t}\right|<k$ or $\left|m_{t}\right|=k$ and $i<n$ for at least one of the letters featuring in $m_{t}$. Applying $\Phi$ to both sides once again, we arrive at the result.

Lemmata 3.1 and 4.2 allow us to construct our spanning set, which we define here.

Definition 4.3. For positive integers $k, n$, let $N S(k, n)$ be the set of standard monomials $m$ which admit a factorisation $m=a u b$, where $a$ is a standard monomial of length $<k$ in $e_{-n}$ and smaller letters, $u$ is a standard monomial in $e_{1-n}, \ldots e_{0}, e_{1}, e_{2}, \ldots, e_{n-1}$, and $b$ is a standard monomial of length $<k$ in $e_{n}$ and bigger letters.

Lemma 4.4. There exist positive integers $k$ and $n$ such that $A$ is spanned by the image of $N S(k, n)$.

Proof. As before, let $k$ be the maximal length of monomials in $g$; that is $k=|g|$.

By Lemmata 3.1 and 4.2 , there exist $n_{1}, n_{2} \in \mathbb{Z}_{\geqslant 1}$ and $\ell_{1}, \ell_{2} \in \mathbb{Z}$ such that for every standard monomial $m=e_{j_{1}} \ldots e_{j_{k}}$, with $j_{1} \leqslant \ldots \leqslant j_{k}$, if $j_{1} \geqslant n_{1}$, then

$$
m=\sum c_{s} m_{s}+h
$$

where $h \in I$, the sum is finite, $c_{s} \in \mathbb{K}$, and the $m_{s}$ are standard monomials such that $i \geqslant \ell_{1}$ for each $e_{i}$ occurring in $m_{s}$, and $i<n_{1}$ for some $e_{i}$ occurring in $m_{s}$; and if $j_{k} \leqslant-n_{2}$, then

$$
m=\sum c_{s} m_{s}+h
$$


where $h \in I$, the sum is finite, $c_{s} \in \mathbb{K}$, and the $m_{s}$ are standard monomials such that $i \leqslant \ell_{2}$ for each $e_{i}$ occurring in $m_{s}$, and $i>-n_{2}$ for some $e_{i}$ occurring in $m_{s}$.

Let $n=\max \left\{n_{1}, n_{2},\left|\ell_{1}\right|,\left|\ell_{2}\right|\right\}$. Repeatedly using the observations above to rewrite $m$ modulo $I$, we obtain the result.

For the rest of the section, let $k, n$ be as given by Lemma 4.4, and let $N S=$ $N S(k, n)$. We call the elements of $N S$ normal words, and a representation of $m \in A$ as a linear combination of (images of) normal words a normal form for $m$, bearing in mind that this normal form may not be unique.

The growth of $N S$ is polynomial, as we next show.

Lemma 4.5. For any positive integer $C$, define a function

$\delta_{C}:\{$ standard monomials in $U(W)\} \longrightarrow \mathbb{N}$

by $\delta_{C}\left(e_{i_{1}} \ldots e_{i_{k}}\right)=\left|i_{1}\right|+\ldots+\left|i_{k}\right|+C$. For any $N \in \mathbb{N}$, let

$$
p_{C}(N)=\#\left\{w \in N S \mid \delta_{C}(w) \leqslant N\right\} .
$$

Then the function $p_{C}$ has polynomial growth.

Proof. The growth of $p_{C}$ does not depend on $C$, so without loss of generality let $C=1$ and let $p=p_{1}$ and $\delta=\delta_{1}$. For a standard monomial $m$, we refer to $\delta(m)$ as the absolute degree of $m$. Note that $U(W)$ is not graded with respect to absolute degree.

Clearly $p(N) \leqslant q(N) r(N)^{2}$, where $q(N)$ is the number of standard monomials in

$$
e_{1-n}, \ldots, e_{0}, e_{1}, \ldots, e_{n-1}
$$

of absolute degree at most $N$, while $r(N)$ is the number of standard monomials in $e_{n}, e_{n+1}, \ldots$ of degree at most $N$ and of length at most $k-1$.

Now, absolute degree, as a function on standard monomials, is always greater than or equal to length. Thus $q(N)$ does not exceed the number of standard monomials in $e_{1-n}, \ldots, e_{n-1}$ of length at most $N$, which is equal to $\left(\begin{array}{c}N+2 n-1 \\ N\end{array}\right) \leqslant c N^{2 n-1}$, for some positive constant $c$ which depends only on $n$ and not on $N$.

On the other hand, in a monomial in $e_{n}, e_{n+1}, \ldots$ of absolute degree at most $N$ and of length at most $k-1$, there are no more than $N$ options for each letter and therefore $r(N) \leqslant N^{k-1}$ for all $N$. Hence $p(N) \leqslant c N^{2 n+2 k-3}$ and has polynomial growth.

\subsection{Choice of filtration}

It remains to estimate the growth of $A$ from the spanning set constructed in the previous subsection. 
Since $A$ is finitely generated, the growth of any finite filtration bounds the growth of $A$. The main result of this subsection is that there is a constant $C$ such that the function $\delta_{C}$ induces a finite filtration of $A$, which by Lemma 4.5 will have polynomial growth.

To have a filtration $A_{1} \subseteq A_{2} \subseteq A_{3} \subseteq \ldots$ on $A=\bigcup A_{i}$ means to choose a map $\rho$ : $A \rightarrow \mathbb{N}$, satisfying

$$
\rho(u v) \leqslant \rho(u)+\rho(v), \quad \rho(u+v) \leqslant \max \{\rho(u), \rho(v)\} \quad \text { and } \quad \rho(\alpha u)=\rho(u),
$$

for any $u, v \in A$ and $\alpha \in \mathbb{K}^{*}$. Suppose that for some $C$, the map $\delta_{C}: N S \rightarrow \mathbb{N}$ has the property that for any two normal words $m_{1}, m_{2} \in N S$, we can find a normal form

$$
\pi\left(m_{1} m_{2}\right)=\sum c_{i} \pi\left(w_{i}\right)
$$

where the $c_{i} \in \mathbb{K}$ and the $w_{i}$ are normal words so that

$$
\delta_{C}\left(w_{i}\right) \leqslant \delta_{C}\left(m_{1}\right)+\delta_{C}\left(m_{2}\right) \text { for all } i
$$

We claim that this is enough to construct the required $\rho$. For, define

$$
\rho(u)=\min _{\substack{\text { normal forms } \\ u=\sum c_{j} \pi\left(w_{j}\right)}}\left\{\max _{j} \delta_{C}\left(w_{j}\right)\right\} .
$$

Then $\rho$ is easily seen to satisfy the required conditions (4.1).

So our goal is to show that there is some constant $C$ so that $\delta_{C}$ satisfies (4.2).

Proposition 4.6. Let $\ell=\max \left\{|i|: e_{i}\right.$ features in $\left.g\right\}$. Then (4.2) holds for $C=$ $4 k^{2} \ell$, where we recall that $k=|g|$.

Proof. It suffices to show that for any normal words $m_{1}, m_{2} \in N S$, and for any normal form $\pi\left(m_{1} m_{2}\right)=\sum c_{i} \pi\left(w_{i}\right)$ for $\pi\left(m_{1} m_{2}\right)$, where $w_{i} \in N S$ and $c_{i} \in \mathbb{K}^{*}$, that

$$
\delta_{0}\left(w_{i}\right) \leqslant \delta_{0}\left(m_{1}\right)+\delta_{0}\left(m_{2}\right)+C \text { for all } i .
$$

So we need to understand how $\delta_{0}$ behaves on the words appearing in a normal form for $\pi\left(m_{1} m_{2}\right)$.

Recall the definition of $n$ from Lemma 4.4. Write

$$
m_{1}=a_{1} u_{1} b_{1} \quad \text { and } \quad m_{2}=a_{2} u_{2} b_{2}
$$

where $u_{1}$ and $u_{2}$ are standard monomials of any length on variables with indices strictly between $-n$ and $n, a_{1}$ and $a_{2}$ are standard monomials of length $<k$ on letters with indices $\leqslant-n$, while $b_{1}, b_{2}$ are standard monomials of length $<k$ on letters with indices $\geqslant n$. Now, normal words are standard monomials, and we first 
use the commutation relations $e_{i} e_{j}=e_{j} e_{i}+(j-i) e_{i+j}$ to write $m_{1} m_{2}$ as a linear combination of standard monomials, that is as a linear combination of words of the form

$$
m_{3}=a_{3} u_{3} b_{3}
$$

where $a_{3}$ is a standard monomial on letters with indices $\leqslant-n, u_{3}$ is a standard monomial on letters with indices strictly between $-n$ and $n$, and $b_{3}$ is a standard monomial on letters with indices $\geqslant n$. Let us call those letters with indexes $|i| \geqslant n$, big letters. Note that in the course of applying the commutation relations, the total number of big letters does not increase. Thus $a_{3}$ and $b_{3}$ have length $\leqslant 2 k-2$, while $u_{3}$ may have any length.

Now we will use the reduction procedure from Lemma 3.1 to see how $\delta_{0}$ changes when we get rid of big letters in $b_{3}$. If the length of $b_{3}$ is less than $k$ we do not have to do anything. Otherwise let $m$ be the monomial composed of the last $k$ letters of $b_{3}$. According to Lemma 3.1, to get rid of one of the (big) letters in $m$, we find a sequence of derivations $D=\partial_{a_{1}} \ldots \partial_{a_{k}}$, with all the $a_{i} \geqslant 1$, and some $c \in \mathbb{K}^{*}$ such that

$$
c D(g)=m+\sum c_{s} m_{s}^{\prime} .
$$

Let $m^{\prime}$ be some $m_{s}^{\prime}$. Now $m^{\prime}$ is a standard monomial which falls into one of three cases:

I. $\left|m^{\prime}\right|<|m|$, and $m^{\prime}$ is obtained from some monomial $\tilde{g}$ in $g$ by applying $D$ and then the commutation relations;

II. $\left|m^{\prime}\right|=|m|$ and $m^{\prime}$ is obtained from some monomial $\tilde{g}$ in $g$ (which necessarily has length $k$ ) by a non-permutational action of $D$;

III. $\left|m^{\prime}\right|=|m|$ and $m^{\prime}$ is obtained from some monomial $\tilde{g}$ in $g$ by a permutational action of $D$.

First, we note what happens to the number of big letters in each case. In case $\mathrm{I}$, since $m^{\prime}$ is shorter than $m$, and all letters in $m$ are big, $m^{\prime}$ contains fewer big letters than $m$. In case II, since the action is non-permutational, there is a letter in $m^{\prime}$ which was present in $\tilde{g}$. But monomials of $g$ consist of letters which are not big, by definition of $n$. Thus the number of big letters in $m^{\prime}$ is smaller than in $m$. In case III, it may be that the number of big letters in $m^{\prime}$ is still equal to $k$, which is the number of (big) letters in $m$, but certainly there are no more than $k=\left|m^{\prime}\right|$ big letters in $m^{\prime}$. Further, by our choice of $n$, in case III all $e_{i}$ occurring in $m^{\prime}$ have $i \geqslant 1$.

We now consider how $\delta_{0}$ changes throughout this process. In situation III, as $m^{\prime} \leqslant m$ and $m^{\prime}$ and $m$ are made of letters $\geqslant e_{1}$, we have $\delta_{0}\left(m^{\prime}\right)=\operatorname{deg}\left(m^{\prime}\right) \leqslant \operatorname{deg}(m)=$ $\delta_{0}(m)$. In cases I and II, $\delta_{0}\left(m^{\prime}\right)$ may be bigger than $\delta_{0}(m)$. Since applying the commutation relations does not increase $\delta_{0}$, we may assume that the monomial $\mathrm{m}^{\prime}$ 
is a (possibly non standard) monomial obtained from applying $D$ to a monomial $\tilde{g}$ of $g$.

Recall that $\bar{g}$ is the $\prec$-leading monomial of $g$, and $m$ is obtained from the monomial $\bar{g}$ by applying $D$. Set $\operatorname{deg}(D)=a_{1}+\ldots+a_{k}$, so applying $D$ increases the degrees of homogenous elements by $\operatorname{deg}(D)$. As $\delta_{0}\left(e_{a+b}\right) \leqslant \delta_{0}\left(e_{a}\right)+\delta_{0}\left(e_{b}\right)$ and all the $a_{i}$ are $\geqslant 1$, we have

$$
\delta_{0}\left(m^{\prime}\right) \leqslant \delta_{0}(\tilde{g})+\operatorname{deg} D
$$

So

$$
\begin{aligned}
\delta_{0}\left(m^{\prime}\right)-\delta_{0}(m) & \leqslant \delta_{0}(\tilde{g})+\operatorname{deg} D-\delta_{0}(m) \\
& =\delta_{0}(\tilde{g})+\operatorname{deg} D-\operatorname{deg} m \quad \text { as letters in } m \text { are big } \\
& \leqslant \delta_{0}(\tilde{g})+|\operatorname{deg} m-\operatorname{deg} D| \\
& =\delta_{0}(\tilde{g})+|\operatorname{deg} \bar{g}| \\
& \leqslant 2 k \ell,
\end{aligned}
$$

by choice of $\ell$, as $\tilde{g}$ and $\bar{g}$ have no more than $k$ letters.

To summarize the discussion above: in cases I and II, we remove at least one big letter and increase $\delta_{0}$ by no more than $2 k \ell$. In case III, we do not remove big letters and do not increase $\delta_{0}$. As proved in Lemma 3.1, after repeating this procedure finitely many times, we may write $m_{1} m_{2}$, modulo $I$, as a linear combination of normal words. However many times we repeat the procedure we remove a maximum of $k-1$ big letters from $b_{3}$, and thus we add a maximum of $2 k(k-1) \ell$ to $\delta_{0}$. Note that in applying this process to $b_{3}$, we never add any letters with indices $<-n$.

After we apply the procedure from Lemma 4.2 to $a_{3}$ at the other end of the word, we have found a normal form for $m_{1} m_{2}$ and have added maximum of another $2 k(k-1) \ell$ to $\delta_{0}$. In other words, we have written

$$
\pi\left(m_{1} m_{2}\right)=\sum c_{i} \pi\left(w_{i}\right)
$$

where the $w_{i}$ satisfy (4.3), as required.

The proof of Theorem 4.1 is now an easy combination of other results in this section.

Proof of Theorem 4.1. Let $C$ be the constant given by Proposition 4.6. The discussion before that proposition shows that setting

$$
A(N)=\operatorname{span}\left\{\pi(w): w \in N S, \pi_{C}(w) \leqslant N\right\}
$$

defines a finite filtration on $A$. By Lemma 4.5, this filtration has polynomial growth, and so $\operatorname{GKdim}(A)<\infty$.

Theorem 4.1 gives the second part of Theorem 1.2, dealing with $U(W)$. 
Remark 4.7. Let $\mathbf{W}_{1}$ be the first Cartan algebra, which is isomorphic to the subalgebra of $W$ spanned by $\left\{e_{n}: n \geqslant-1\right\}$. This is a simple graded Lie algebra of polynomial growth. Similar methods to those used in Theorem 4.1 show that $U\left(\mathbf{W}_{1}\right)$ has just infinite GK-dimension. To show this for all simple graded Lie algebras of polynomial growth is the subject of ongoing work.

\section{Central quotients of the enveloping and symmetric algebras of $\mathrm{Vir}$}

In this section we first prove that all the central quotients $U(\operatorname{Vir}) /(c-\varkappa)$ have just infinite GK-dimension, completing the proof of Theorem 1.2, and then consider the related Poisson algebras $S(\operatorname{Vir}) /(c-\varkappa)$. Because the ideas of the proofs are similar to those in previous sections, we leave some details to the reader.

\subsection{Central quotients of the enveloping algebra of Vir}

Fix $\varkappa \in \mathbb{K}$ and let $R=U(\operatorname{Vir}) /(c-\varkappa)$. Essentially the same argument as for the full Witt algebra works to show that $R$ has just infinite GK-dimension; we give a sketch of the proof.

Note that $R$, like $U(W)$, has a basis of standard monomials in the $e_{i}$.

Definition 5.1. For positive integers $k, n$, let $N S(k, n) \subset R$ be the set of standard monomials $m$ in the $e_{i}$ which admit a factorisation $m=a u b$, where $a$ is a standard monomial of length $<k$ in $e_{-n}$ and smaller letters, $u$ is a standard monomial in $e_{1-n}, \ldots e_{0}, e_{1}, e_{2}, \ldots, e_{n-1}$, and $b$ is a standard monomial of length $<k$ in $e_{n}$ and bigger letters.

Lemma 5.2. Let $g$ be a nonzero element of $R$, let $I=(g)$, and let $A=R / I$. There exist positive integers $k$ and $n$ such that $A$ is spanned by the image of $N S(k, n)$.

Proof. The key point is that the reduction formulae in Lemmata 3.1 and 4.2 still hold. As before, let $\partial_{a}=\left[\ldots, e_{a}\right]$ as a linear operator on $R$ and consider the effect of applying some $\partial_{a_{1}} \ldots \partial_{a_{k}}$ to a standard monomial of length $k$. If $\varkappa \neq 0$ and some expression of the form $\left[e_{-a_{j}}, e_{a_{j}}\right]$ has been computed, we may obtain some standard monomials of length $<k$ in the result; but the leading term will have length $k$ and will be given by the procedures in the previous section. Thus the proof of Lemma 4.4 goes through in this situation, almost without change.

Theorem 5.3. Let $I=(g)$ be a two-sided ideal in $R$ generated by one nonzero element $g \in R$. Then $A=R / I$ has polynomial growth. 
Proof. We may define the functions $\delta_{C}$ just as with $U(W)$. The only part of the argument which is different for $R$ is the proof of Proposition 4.6. When we compute

$$
c \partial_{a_{1}} \ldots \partial_{a_{k}}(g)=m+\sum_{s} c_{s} m_{s}^{\prime}
$$

as in (4.4), consider some $m^{\prime}=m_{s}^{\prime}$ as before. In addition to cases I, II, III as in the proof of Proposition 4.6, we may have

I'. $\left|m^{\prime}\right|<|m|$, and $m^{\prime}$ is obtained from some monomial $\tilde{g}$ in $g$ by applying $\partial_{a_{1}} \ldots \partial_{a_{k}}$ and then applying the relation

$$
\left[e_{-i}, e_{i}\right]=2 i e_{0}+\frac{i-i^{3}}{12} \delta_{i+j, 0} \varkappa .
$$

As before, applying this relation does not increase $\delta_{0}$, so we may assume that $\mathrm{m}^{\prime}$ is a (possibly non standard) monomial obtained from applying $D$ to a monomial $\tilde{g}$ of $g$. The argument of Proposition 4.6 goes through with only minor changes, and the result follows just as in the proof of Theorem 4.1.

Theorem 5.3 completes the proof of Theorem 1.2 by establishing the part dealing with $U($ Vir $)$.

Remark 5.4. By the same method as in the proof of Theorem 5.3, one may show that the localised enveloping algebra $U(V i r) \otimes_{\mathbb{K}[c]} \mathbb{K}(c)$, considered as an algebra over $\mathbb{K}(c)$, has just infinite GK-dimension. It follows, using a similar argument to the proof of [KL00, Lemma 3.10], that $U(V i r) \otimes_{\mathbb{K}[c]} \mathbb{K}(c)$ has just infinite GK-dimension considered as a $\mathbb{K}$-algebra. We leave the details to the reader.

\subsection{Central quotients of the symmetric algebra of $\mathrm{Vir}$}

In this subsection, let $\varkappa \in \mathbb{K}$ and let $R=S(W) /(c-\varkappa)$. Since $c-\varkappa$ is Poisson central, $R$ is a Poisson algebra; in fact if we filter $U($ Vir $)$ by setting $\left|e_{i}\right|=1$ and $|c|=0$, then $R$ is the associated graded ring of $U(\operatorname{Vir}) /(c-\varkappa)$. Note that if we define $d_{a}=\left\{\ldots, x_{a}\right\}$ and $\partial_{a}=\left[\ldots, e_{a}\right]$ as before, then (3.2) still holds.

Similar arguments to those that have gone before prove:

Theorem 5.5. Let $g$ be a nonzero element of $R$ and let $I=\{(g)\}$ be the Poisson ideal generated by $g$. Then $A=R / I$ has polynomial growth.

Proof. The reduction process works as before: writing $g=\operatorname{gr}\left(g^{\prime}\right)$ and computing

$$
h=c \partial_{a_{1}} \ldots \partial_{a_{k}}\left(g^{\prime}\right)=m+\sum_{s} c_{s} m_{s}^{\prime}
$$


as in $(5.1)$, by $(3.2)$

$$
\operatorname{gr}(h)=\operatorname{gr}(m)+\sum_{t} c_{t} \operatorname{gr}\left(m_{t}^{\prime}\right),
$$

where the only $m_{t}^{\prime}$ surviving have length $k$. Thus as in Lemma 5.2 there are $k$ and $n$ so that $A$ is spanned by the image of $\operatorname{gr}(N S(k, n))$.

Comparing $\delta_{0}\left(\operatorname{gr}\left(m^{\prime}\right)\right)$ with $\delta_{0}(\operatorname{gr}(m))$ as in the proof of Proposition 4.6 we see that only cases II and III occur. The conclusion of Proposition 4.6 still holds, and so as in the proof of Theorem $4.1 \operatorname{GKdim}(A)<\infty$.

Remark 5.6. Similarly, one may show that $S\left(\mathbf{W}_{1}\right)$ has just infinite GK-dimension. We omit the proof.

\section{Applications}

In this section we give several applications of Theorem 1.2. We first give a short proof that Verma modules for Vir are faithful over the appropriate central factor of $U(V i r)$. (A more direct proof is an unpublished result of Nolan Wallach [WS13].) We next prove that $U\left(W_{+}\right), U(W)$, and $U(V i r)$ all satisfy the ascending chain condition on completely prime ideals. As a consequence, these algebras are Hopfian: they are not isomorphic to any proper quotient.

\subsection{Annihilators of induced modules}

Fix $\lambda, \varkappa \in \mathbb{K}$. Note that the Virasoro algebra Vir has a triangular decomposition: define $\mathfrak{n}_{+}:=\mathbb{K}\left(e_{n}: n \geqslant 1\right), \mathfrak{h}:=\mathbb{K}\left(c, e_{0}\right)$, and $\mathfrak{n}_{-}:=\mathbb{K}\left(e_{n}: n \leqslant-1\right)$. Let $\mathfrak{b}_{+}:=$ $\mathfrak{n}_{+} \oplus \mathfrak{h}$. Let $\mathbb{K}_{\varkappa, \lambda}$ be the one-dimensional representation of $\mathfrak{b}_{+}$where $\mathfrak{n}_{+}$acts trivially, $c$ acts as $\varkappa$, and $e_{0}$ acts as $\lambda$. Then define the Verma module $M_{\varkappa, \lambda}$ to be $U(\operatorname{Vir}) \otimes_{U\left(\mathfrak{b}_{+}\right)} \mathbb{K}_{\varkappa, \lambda}$. It is immediate that

$$
M_{\varkappa, \lambda} \cong U(\operatorname{Vir}) / U(\operatorname{Vir})\left(c-\varkappa, e_{0}-\lambda, e_{n}: n \geqslant 1\right)
$$

and that $M_{\varkappa, \lambda}$ is non-positively graded, with $\operatorname{dim}\left(M_{\varkappa, \lambda}\right)_{-n}=\mathcal{P}(n)$, the $n$ 'th partition number.

Verma modules are examples of the larger class we call, slightly imprecisely, induced modules. These are modules of the form $M=U(\operatorname{Vir}) \otimes_{U\left(\mathfrak{b}_{+}\right)} M^{\prime}$, where $M^{\prime}$ is a representation of $\mathfrak{b}_{+}$. Besides Verma modules, examples include logarithmic representations, where $\operatorname{dim}_{\mathbb{K}} M^{\prime}<\infty$ and where $\mathfrak{n}_{+}$acts trivially on $M^{\prime}, c$ acts as a scalar, and $e_{0}$ acts as a non-semisimple matrix. These representations are important in logarithmic conformal field theory, see [GK96]. 
Whittaker modules [OW09] form another class of examples. Here let $\mathfrak{n}^{\prime}=\mathfrak{n} \oplus \mathbb{K} c$ and let $M^{\prime \prime}$ be the one-dimensional $\mathfrak{n}^{\prime}$ module where $c$ acts as a scalar, and the $e_{n}$ act trivially for $n \geqslant 3$. The module

$$
M=U(V i r) \otimes_{U\left(\mathfrak{n}^{\prime}\right)} M^{\prime \prime} \cong U(V i r) \otimes_{U\left(\mathfrak{b}_{+}\right)} U\left(\mathfrak{b}_{+}\right) \otimes_{U\left(\mathfrak{n}^{\prime}\right)} M^{\prime \prime}
$$

is a Whittaker module. If $e_{1}, e_{2}$ act nontrivially on $M^{\prime \prime}$, then $M$ is simple by [OW09, Corollary 4.5]. All of these examples are annihilated by some $c-\varkappa$, where $\varkappa \in \mathbb{K}$, and so have central character $\varkappa$.

Using Theorem 1.2, we may immediately compute the annihilator of an induced module.

Theorem 6.1. Let $M^{\prime}$ be a representation of $\mathfrak{b}_{+}$with central character $\varkappa \in \mathbb{K}$. Let $M=U(\operatorname{Vir}) \otimes_{U\left(\mathfrak{b}_{+}\right)} M^{\prime}$. Then $\operatorname{Ann}_{U(\text { Vir })} M=(c-\varkappa)$.

Proof. Clearly $(c-\varkappa) M=0$.

Let $\mathcal{P}$ be the set of negative partitions $\underline{\lambda}=\left(\lambda_{1}, \ldots, \lambda_{k}\right)$ where the $\lambda_{i}$ are negative integers with $\lambda_{1} \leqslant \lambda_{2} \leqslant \ldots \leqslant \lambda_{k}$. If $\underline{\lambda}=\left(\lambda_{1}, \ldots, \lambda_{k}\right) \in \mathcal{P}$, let $e_{\underline{\lambda}}=e_{\lambda_{1}} \ldots e_{\lambda_{k}}$. If $0 \neq m \in M^{\prime}$, it follows from the Poincaré-Birkhoff-Witt theorem that the elements $\left\{e_{\underline{\lambda}} \otimes m: \underline{\lambda} \in\right.$ $\mathcal{P}$ \} are linearly independent in $M$, and thus, as these elements are in bijection with partitions, that $M$ has subexponential growth and infinite Gelfand-Kirillov dimension.

Let $K=\operatorname{Ann}_{U(V i r)} M$. If $K \supseteq(c-\varkappa)$ then by Theorem $1.2 U($ Vir $) / K$ has polynomial growth and thus, by [KL00, Proposition 5.1(d)], so does $M$. This contradiction shows that $K=(c-\varkappa)$.

Corollary 6.2. For any $\varkappa, \lambda \in \mathbb{K}$, the Verma module $M_{\varkappa, \lambda}$ is a faithful $U(\operatorname{Vir}) /(c-\varkappa)$-module.

Corollary 6.2 is an unpublished result of Nolan Wallach [WS13], and is independently due to Olivier Mathieu in unpublished work; see [CM07, footnote 2, p. 496]. We thank Rupert Wei Tze Yu for pointing out this reference to us.

It is known [FF84, Theorem 1.2] that for any $\varkappa$, the module $M_{\varkappa, \lambda}$ is simple for generic $\lambda$. Thus it follows immediately that $U(\operatorname{Vir}) /(c-\varkappa)$ is primitive.

Corollary 6.3. Let $N$ be a logarithmic representation or a Whittaker module over Vir. Then $\operatorname{Ann}_{U(\text { Vir })}(N)=(c-\varkappa)$ for some $\varkappa \in \mathbb{K}$.

\subsection{Completely prime ideals}

In [PS17, Conjecture 1.3], it is conjectured that $U\left(W_{+}\right)$satisfies the ascending chain condition on two-sided ideals. We cannot prove this, but we do show 
Proposition 6.4. The algebras $U\left(W_{+}\right)$and $U(V i r)$ satisfy the ascending chain condition $(A C C)$ on completely prime ideals.

Proof. We first note that any ring $R$ of finite or just infinite GK-dimension satisfies ACC on completely prime ideals. Letting $P_{0}$ be the first ideal in the chain, it is sufficient to show that $R / P_{0}$ has ACC on completely prime ideals. Thus we may replace $R$ by $R / P_{0}$ and assume that $R$ is a domain with GKdim $R<\infty$. Now if $I$ is a nonzero ideal of $R$, then by [KL00, Proposition 3.15], GKdim $R / I \leqslant \operatorname{GKdim} R-1$, so by induction the length of a chain of completely prime ideals is bounded by $\operatorname{GKdim} R$. Thus by Theorem $1.2, U\left(W_{+}\right)$and $U(\operatorname{Vir}) /(c-\varkappa)$ (for any $\varkappa \in \mathbb{K}$ ) have ACC on completely prime ideals.

In fact, note that if $f[x] \in \mathbb{K}[x]$ is irreducible, then $U(\operatorname{Vir}) /(f(c))$ has just infinite GK-dimension and thus ACC on completely prime ideals. To see this, let $\mathbb{K}^{\prime}$ be the extension field $\mathbb{K}[x] / f(x)$ of $\mathbb{K}$, and note that

$$
U(\operatorname{Vir}) /(f(c))=U_{\mathbb{K}}(\operatorname{Vir}) /(f(c)) \cong U_{\mathbb{K}^{\prime}}(\operatorname{Vir}) /(c-x) .
$$

This last has just infinite GK-dimension by Theorem 1.2.

We now consider an ascending chain $P_{1} \subseteq P_{2} \subseteq \ldots$ of completely prime ideals of $U($ Vir $)$. If $\bigcup P_{n}$ contains a nonzero element of $\mathbb{K}[c]$, then as the $P_{n}$ are prime and $c$ is central, some $P_{n}$ contains an irreducible polynomial $f(c) \in \mathbb{K}[c]$. By the first part of the proof, therefore, the chain stabilizes.

So we may assume that each $P_{n} \cap \mathbb{K}[c]=0$. As $P_{n}$ is prime, each $U(\operatorname{Vir}) / P_{n}$ is $\mathbb{K}[c]$-torsionfree. Thus if $P_{n} \neq P_{n+1}$, then $\left(P_{n+1} / P_{n}\right) \otimes_{\mathbb{K}[c]} \mathbb{K}(c) \neq 0$ and so

$$
P_{n} \otimes_{\mathbb{K}[c]} \mathbb{K}(c) \neq P_{n+1} \otimes_{\mathbb{K}[c]} \mathbb{K}(c) .
$$

Further, these ideals are completely prime as

$$
U(V i r) \otimes_{\mathbb{K}[c]} \mathbb{K}(c) / P_{n} \otimes_{\mathbb{K}[c]} \mathbb{K}(c) \cong\left(U(V i r) / P_{n}\right) \otimes_{\mathbb{K}[c]} \mathbb{K}(c)
$$

is a domain.

Thus it suffices to show that $U(V i r) \otimes_{\mathbb{K}[c]} \mathbb{K}(c)$ has ACC on completely prime ideals. By Remark 5.4, U(Vir $) \otimes_{\mathbb{K}[c]} \mathbb{K}(c)$ has just infinite GK-dimension. Thus by the first part of the proof, $U(V i r) \otimes_{\mathbb{K}[c]} \mathbb{K}(c)$ satisfies the ACC on completely prime ideals.

\subsection{The Hopfian and Bassian properties}

To end the paper, we consider two ring-theoretic properties which are related to noetherianity. A ring $R$ is Hopfian if $R$ is not isomorphic to any proper quotient 
$R / J$ (equivalently, any epimorphism from $R \rightarrow R$ is an isomorphism). More strongly, $R$ is Bassian if there is no injection of $R$ into any proper quotient $R / J$. We thank Lance Small for introducing us to these concepts.

Proposition 6.5. The algebras $U\left(W_{+}\right), U(W), U\left(\mathbf{W}_{1}\right)$, and $U(\operatorname{Vir}) /(c-\varkappa)$ are Bassian and Hopfian, and U(Vir) is Hopfian.

That $U\left(W_{+}\right)$is Hopfian is proved in [RS17, Remarks 2.2], and [RS17, Section 4] asks whether $U(W)$ is Bassian or Hopfian.

Proof. If $R$ has just infinite GK-dimension, then $\operatorname{GKdim} R / J<\operatorname{GKdim} R$ for any proper ideal $J$ of $R$, so $R$ cannot inject in $R / J$. Thus the Bassian (and thus Hopfian) property for $U\left(W_{+}\right), U(W)$, and $U(\operatorname{Vir}) /(c-\varkappa)$ follows from Theorem 1.2. For $U\left(\mathbf{W}_{1}\right)$ it follows from Remark 4.7.

To show that $U($ Vir $)$ is Hopfian, let $R=U($ Vir $)$ and let $f$ be a surjective endomorphism of $R$, with kernel $J$. As $R / J \cong \operatorname{Im}(f)$ is torsionfree as a module over $\mathbb{K}[c]$, the complex

$$
0 \longrightarrow J \otimes_{\mathbb{K}[c]} \mathbb{K}(c) \longrightarrow R \otimes_{\mathbb{K}[c]} \mathbb{K}(c) \longrightarrow(R / J) \otimes_{\mathbb{K}[c]} \mathbb{K}(c) \longrightarrow 0
$$

is exact. Now by Remark 5.4, we must have $J \otimes_{\mathbb{K}[c]} \mathbb{K}(c)=0$. As $R$ is $\mathbb{K}[c]$-torsionfree, $J=0$.

That $U(V i r)$ is Hopfian also follows from [RS17, Corollary 2.6] and Proposition 6.4. We do not know whether $U(V i r)$ is Bassian.

Acknowledgments. This work is funded by the EPSRC grant EP/M008460/1/. The first named author is grateful to IHES and MPIM, where part of this work was done, for hospitality and support.

We thank José Figueroa-O'Farrill, Tom Lenagan, and Lance Small for useful comments and discussions.

\section{References}

[AS74] Amayo, R. K. and Stewart, I., Infinite-dimensional Lie algebras, Noordhoff International Publishing, Leyden, 1974.

[Bro07] Brown, K. A., Noetherian Hopf algebras, Turkish J. Math. 31 (2007), 7-23.

[CM07] Conley, C. H. and Martin, C., Annihilators of tensor density modules, J. Algebra 312 (2007), 495-526.

[FF84] FeIGgin, B. L. and Fuchs, D. B., Verma modules over the Virasoro algebra, in Topology (Leningrad, 1982), Lecture Notes in Math. 1060, pp. 230-245, Springer, Berlin, 1984. 
[GK96] Gabardie, M. R. and Kausch, H. G., Indecomposable fusion products, $N u$ clear Phys. B 477 (1996), 293-318.

[KKM83] Kirillov, A. A., Kontsevich, M. L. and Molev, A. I., Algebras of intermediate growth, Akad. Nauk SSSR Inst. Prikl. Mat. Preprint (1983), 19. Translated in Selecta Math. Soviet. 9 (1990), 137-153.

[KL00] Krause, G. R. and Lenagan, T. H., Growth of algebras and Gelfand-Kirillov dimension, revised ed., Graduate Studies in Mathematics 22, American Mathematical Society, Providence, RI, 2000.

[Mat92] Mathieu, O., Classification of simple graded Lie algebras of finite growth, Invent. Math. 108 (1992), 455-519.

[OW09] Ondrus, M. and Wiesner, E., Whittaker modules for the Virasoro algebra, $J$. Algebra Appl. 8 (2009), 363-377.

[PS17] Petukhov, A. and Sierra, S. J., Ideals in the enveloping algebra of the positive Witt algebra, 2017. arXiv: 1710.10029.

[RS17] Rowen, L. and Small, L., Hopfian and Bassian algebras, 2017. arXiv: 1711.06483.

[Smi76] Sмiтh, M. K., Universal enveloping algebras with subexponential but not polynomially bounded growth, Proc. Amer. Math. Soc. 60 (1976), 22-24. 1977.

[SW14] Sierra, S. J. and Walton, C., The universal enveloping algebra of the Witt algebra is not noetherian, Adv. Math. 262 (2014), 239-260.

[Ufn78] UfnarovskiI, V. A., The growth of algebras, Vestnik Moskov. Univ. Ser. I Mat. Mekh. 4 (1978), 59-65.

[WS13] Wallach, N. and Small, L., 2013. Personal communication.

Natalia K. Iyudu

School of Mathematics

The University of Edinburgh

Edinburgh

GB-EH93FD

United Kingdom

n.iyudu@ed.ac.uk

Received October 17, 2019

in revised form February 21, 2020

\author{
Susan J. Sierra \\ School of Mathematics \\ The University of Edinburgh \\ Edinburgh \\ GB-EH93FD \\ United Kingdom \\ s.sierra@ed.ac.uk
}

